vomiting occurred. Bowels al ways regular. A week ago she had another fit, leaving an apparent palsy of all the eye muscles. Vomiting took place occasionally. I was called in to see the child, as it was supposed that symptoms, \&c., were due to a tumour near the quadrigeminal bodies.

State.-The child in poor general condition, lying in bed on right side, and complaining of localised pain over left occipital region which had been present for four days. Mental condition quite clear, but speaks in a monotone and answers quickly and rationally. The right hand is held in left hand, and moved about by latter. Head measurement $50 \frac{1}{2} \mathrm{~cm}$., regnlar in shape. Examination of cranial nerves: 1 . Smell sense delicate-not the slightest impairment. 2. No change in optic nerves and fundi ; fields of vision normal. Vision normal. 3, 4,6. Right side: Pupil dilated and movements in all directions impaired; moves more than left. Slight exophthalmos. Left side: Eye apparently immovable, but on persuasion child moved eye slightly in all directions. Reaction to light good; reaction to accommodation could not be determined. 5. No loss of sensation. 7. Supranuclear lesion on right side; left side apparently normal. 8. Hearing normal on both sides. 9. Taste normal. 11. Unaffected. 12. Deviation to right side when tongue is protruded.

Right arm: Kept flexed over chest. Some active flexion and extension of elbow and distal joints. Grasps hand well but weakly. Tendon reflexes absent. No loss of sensation. Arm, forearm, and hand muscles definitely atrophied. Left arm: Loss of tendon reflexes, but otherwise normal in all its.movements, not showing an intention tremor. Trunk: Normal sensation. Abdominal reflexes right side are absent, but are brisk on left side. Sphincters normal. Right lower limb: Definite paresis compared with left. Knee-jerk absent; other tendon-jerks also absent. No ankle clonus. Hallux erectus present. Definite disuse atrophy present; limb is $2.5 \mathrm{~cm}$. shorter than its fellow. No loss of sensation. Left lower limb: Stronger than right. Movements well coördinated. Knee-jerk and other tendon-jerks absent. Ankle clonus absent. Babinski's sign: Extensor response. No scoliosis. Definite contracture of post-vertebral muscles in occipital region. Wassermann reaction negative (two oceasions). Cerebro-spinal fluid not examined. Urine normal. T. about $99^{\circ}$ F. at night. P. 120 . Heart, lungs, and abdominal viscera normal.

Fits, intense headache, occasional vomiting, and the eye symptoms suggested intracranial pressure, but of this there was no evidence on further investigation. Friedreich's disease, with which much of the condition agrees, is excluded by the absence of definite ataxia and of hereditary transmission.

Buenos Aires.

\section{PETROLEUM IN THE TREATMENT OF PYORRHEA ALVEOLARIS.}

\section{BY H. J. CORIN, L.D.S.}

THE treatment of pyorrhœa alveolaris should be undertaken by the dentist only in conjunction with the patient's medical adviser, since pyorrhcea is now commonly acknowledged to be aggravated by, if not actually due to, causes other than dental. It is, for instance, in many cases primarily induced by the absorption of toxins from a septic small intestine, a condition which obviously needs skilled medical attention.

For such a condition purified petroleum is used widely and successfully, both in those cases where surgical interference is necessary, and also in those more common instances where no operation is required. Most probably the therapeutic action of petroleum is known to the vast majority of medical men; but I would like to emphasise the caution given some time ago in this journal after various investigations, ${ }^{1}$ that only the purest oil should be administered.

In a case where there was only a slight discharge of pus from the gums, one course of local treatment, the use of an effective mouth-wash and the patient ordered to take petroleum emulsion, produced a complete cure. The patient was unable to come to town for six weeks after treatment, but seen at the end of this period there was absolutely no trace of discharge.

For long-standing cases where there is a large discharge I have found petroleum with guaiacol to give wonderful results in clearing up the conditions quickly, and when there is acute gingivitis petroleum with salol is useful. After my experience I can confidently recommend this method of treatment as effective in many instances where other methods have failed.

old Burlington-street, $w$.

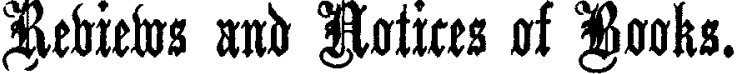

Organio to Human: Psychological and Sooial. By HeNRT Ma Udslex, M.D. Lond. London : Macmillan and Co., Limited. 1916. Pp. 386. Price 12s, net.

THIS latest work by Dr. Maudsley is fully worthy of the high reputation that has for long placed him in the front rank of contemporary scientific thinkers and writers on mental processes in health and disease. His first book, the "Physiology and Pathology of Mind," published hard on half a century ago, showed his mastery of all then available knowledge of the structure and functions of the human nervous system, no less than his signal capacity for thinking scientifically. It contained, moreover, the germs of much that its author has since from time to time given to us in his well-known works expanded and illustrated by further experience and mature reflection. Even as Dr. Maudsley's earliest work drew the attention of philosophical thinkers more than that of practising medical men, so probably will this latest book appeal mainly to the scientific student of psychology and sociology whether without or within the ranks of the medical profession: It must, indeed, be considered chiefly as an important contribution to the still vexed controversy between the warring schools of "materialists" or "mechanists" on the one hand, and of "vitalists" or "spiritualists" on the other. Justly pre-eminent though Dr. Maudsley is as an authority on the subject of insanity, and much as his other work owes to his practical knowledge and experience in this special study, his most enduring title to fame will be that of the scientific philosopher who in his day and generation consistently and persistently upheld the principle of the unity of the human organism and its continuity with the rest of Nature's processes.

"A true psychology" (says Dr. Maudsley in the first chapter of the book before us) "must be founded on a clear and distinct recognition of the essential continuity and constant flux of things in which all so-called causes are effects and all so-called effects causes: mind be conceived in thought as a part of the nature which its organisation is in fact. Continuity of motion without end, the perpetual flux which such old Greek philosophers as Democritus, Heraclitus, and Epicurus insisted on, and Lucretins poetically expounded, that is the fundamental fact: the world never the same for an instant, nor two minds ever the same, nor any mind ever the same for a single hour."

Again :-

"Into the composition of every idea, of every mood, of every act, of every reflection, every organ and every gland of the body has entered essentially; the minutely different structures and physico-chemical changes and exchanges of the parts in different persons producing different temperaments, and the signally special organs of male and female bodies different mental qualities requiring their special direction of development and function. To deride the notion of mechanism as anywise applicable to life and life in mind is the natural error of him who, ignorant of the exquisitely subtile physical and chemical precesses at work in the simplest vital substance, thinks grossly only of inert matter and its mechanism as exemplified in the structure of a wheel-barrow or a steam-engine."

One more quotation must suffice for the purpose of indicating some of the main lines of thought in this book :-

"That all positive intelligence is sense-evolved, though true, is not the whole truth: motor reactions contribute as essentially as sensory impressions to the construction of a mental organisation and fitly respond to them in the complex composition of ideas and judgments. The old saying, Nihil in intellectu quod non prius in sensu might It is clearly apparent that no adequate criticism of the main arguments of this book is possible within the limits of this notice. Even to attempt it would involve a useless plunge into a troubled sea of dispute. But this much may be said in some justification of the high degree of value which we attribute to it. The least biassed thinker on matters psychological, who is dissatisfied with any theory concerning mental phenomena which involves the importation of a purely imagined and unverifiable agency in explanation, but still may find some difficulty in regarding such phenomena as natural products of organisation, is at least likely to discover, after carefully studying Dr. Maudsley's book, that part, if not all, of his difficulty is lessened or removed. The case for 